S0125

\section{Epigenetic pathways in PTSD}

\section{T. Klengel}

Department Of Psychiatry, McLean Hospital and University Medical Center Goettingen, Belmont, United States of America doi: $10.1192 /$ j.eurpsy.2021.120

Abstract Body: Nonhuman primates (NHPs) are critical for translational research due to their close genetic, physiological, and behavioral similarity to humans. In particular, higher brain functions depend on brain regions and neural circuits that evolved differently between primates and rodents. Thus, NHPs are a strong translational model system to investigate the pathophysiology and relevant biological correlates of mental disorders. This talk will focus on translational approaches leveraging NHP models to advance our understanding of environmentally induced epigenetic changes in post-traumatic stress disorder (PTSD). Environmental factors including early life stress significantly contribute to risk and resilience for psychiatric disorders including PTSD. However, human studies are often confounded, and it remains challenging to identify robust epigenetic signals in clinical populations even in large studies. We investigate the natural spectrum of behavioral phenotypes in rhesus macaques to complement human studies with a focus on stress and fear. This talk will present data on epigenetic signatures of fear and the effects of early life stress in rhesus monkeys and their relationship to human studies.

Disclosure: No significant relationships.

\section{New perspectives on bipolar disorder}

\section{S0121}

\section{New insights on affective morbidity and childhood maltreatment}

\section{Kumari}

Life Sciences, Brunel University London, Uxbridge, United Kingdom doi: 10.1192/j.eurpsy.2021.121

Childhood maltreatment (physical, sexual or emotional abuse, and physical or emotional neglect, by a parent, caregiver or other adult) is a major problem of global significance. Children who suffered maltreatment of any kind are known to experience a range of mental health problems, including depression, anxiety, psychosis, substance abuse, eating disorders, suicidal symptomatology and personality disorder. These problems often emerge in childhood and last through adulthood to old age. Furthermore, people with a history of childhood maltreatment show a worse-than-usual response to standard treatment approaches to improve their mental health. There is growing evidence that emotional abuse, one of the most prevalent forms of childhood maltreatment, may have the most wide-ranging impact of all maltreatment types on mental health outcomes as it appears to be a transdiagnostic risk factor for several psychiatric disorders, and found to me more strongly associated with development of affective disorders than physical abuse or neglect. There is a need to move beyond simple association studies and shift the research focus on sophisticated multimodal studies to fully understand the psychobiological mechanisms underlying affective morbidity, as well as the protective factors that might promote resilience, in the face of (specific forms of) childhood maltreatment.

Disclosure: No significant relationships.

Keywords: emotional abuse; Neglect; physical abuse

\section{S0122}

\section{The clinical relevance of childhood manic symptoms}

\section{S. Frangou}

Pychiatry, Icahn School of Medicine at Mount Sinai, New York City, United States of America

doi: 10.1192/j.eurpsy.2021.122

Background: The Adolescent Brain and Cognitive Development (ABCD) study, a US population-based sample of 10 year-olds, offers a unique opportunity to examine the neural correlates of manic-like symptoms presenting in children about to enter adolescence.

Methods: The study will avail of the rich dataset of over 11,000 children aged 9-10 years at enrolment using data from the baseline and 2-year follow-up assessment. The analyses aim to track the evolution of manic-like symptoms between the two follow-up waves and test their sensitivity of their association with brain correlates.

Results: Data analyses are ongoing and will focus on changes in manic-like symptoms, focusing on youth with remitting, persistent and emerging symptoms and examine their associations with brain structure and resting-state functional connectivity.

Conclusions: The results will inform about the early trajectory of manic-like symptoms and offer new insights into their brainrelated correlates.

Disclosure: No significant relationships.

Keywords: childhood mania

\section{Early intervention in psychoses: Assessment and treatment to improve functional outcome}

\section{S0125}

\section{Prediction of drop-out and functional impairment in recent-onset schizophrenia spectrum disorders}

A. Mucci $^{1 \star}$, P. Bucci ${ }^{1}$, I. Winter Van Rossum ${ }^{2}$, C. Arango ${ }^{3}$, L. Baandrup ${ }^{4}$, B. Glenthøj ${ }^{4}$, P. Dazzan ${ }^{5}$, A. Demjaha ${ }^{5}$, P. Mcguire ${ }^{6}$, C. Martínez Díaz-Caneja ${ }^{7}$, S. Leucht ${ }^{8}$, R. Rodriguez-Jimenez ${ }^{9}$, R. Kahn ${ }^{10}$ and S. Galderisi

${ }^{1}$ Department Of Psychiatry, Univeristy of Campania Luigi Vanvitelli, Naples, Italy; ${ }^{2}$ Department Of Psychiatry, University Medical Center Utrecht Brain Center, Utrecht University, Utrecht, Netherlands; ${ }^{3}$ Child And Adolescent Department Of Psychiatry, Hospital General Universitario Gregorio Marañón, Madrid, Spain; ${ }^{4}$ Department Of Clinical Medicine, University of Copenhagen, Copenhagen, Denmark; ${ }^{5}$ Department Of Psychosis Studies, Institute of Psychiatry, Psychology and Neuroscience, King's College, London, United Kingdom;

${ }^{6}$ Department Of Psychosis Studies, King's College London, London, United Kingdom; ${ }^{7}$ Psychiatry, Hospital General Universitario Gregorio Marañón, Madrid, Spain; ${ }^{8}$ Department Of Psychiatry And Psychotherapy, School of Medicine, Technical University Munich, 
Munich, Germany; ${ }^{9}$ Psychiatry, 12 de Octubre Hospital Research Institute, CIBERSAM, Madrid, Spain and ${ }^{10}$ Psychiatry, The Mount Sinai Hospital, New York, United States of America

${ }^{*}$ Corresponding Author.

doi: 10.1192/j.eurpsy.2021.123

Persistent negative symptoms are associated with worse outcome in both first-episode and chronic subjects with schizophrenia. The identification of these symptoms in recent-onset subjects is still controversial as retrospective data are often unavailable. The prospective assessment of persistence of negative symptoms might represent a valid alternative but the length of the persistence is still to be established. The present study investigated the prevalence of negative symptoms of moderate severity, unconfounded by depression and extrapyramidal symptoms at baseline in a large cohort of patients in the early stage of a schizophrenia-spectrum disorder, recruited to the OPTiMiSE trial. Persistent unconfounded negative symptoms were assessed at 4, 10 and 22 weeks of treatment. Symptomatic remission, attrition rate and psychosocial functioning was evaluated in subjects with short-term (4 weeks) persistent negative symptoms (PNS) and in those with negative symptoms that did not persist at follow-up and/or were confounded at baseline (N-PNS). Negative symptoms of moderate severity were observed in $59 \%$ of subjects at baseline and were associated to worse global functioning. PNS were observed in $7.9 \%$ of the cohort, unconfounded at both baseline and end of 4-week treatment. PNS subjects showed lower remission and higher attrition rates at the end of all treatment phases. Fifty-six percent of subjects completing phase 3 (clozapine treatment) had PNS, and $60 \%$ of them were non-remitters at the end of this phase. The presence of short-term PNS during the first phases of psychosis was associated with poor clinical outcome and resistance to antipsychotic treatment, including clozapine.

Disclosure: Prof Mucci has been a consultant and/or advisor to or has received honoraria from Gedeon Richter Bulgaria, Janssen Pharmaceuticals, Lundbeck, Otsuka, Pfizer and Pierre Fabre.

Keywords: First-episode schizophrenia; Functional Outcome; Persistent negative symptoms

\section{S0126}

Negative symptoms assessment in early intervention settings: Implications for early identification and treatment

\author{
L.B. Glenthøj ${ }^{1 \star}$, M. Nielsen ${ }^{2}$ and M. Nordentoft ${ }^{1}$ \\ ${ }^{1}$ Copenhagen Research Centre On Mental Health, Mental Health \\ Centre Copenhagen, University of Copenhagen, copenhagen, \\ Denmark and ${ }^{2}$ Centre For Clinical Intervention And Neuropsychiatric \\ Schizophrenia Research, Cins, Glostrup, Denmark, Mental Health \\ Centre Glostrup, Glostrup, Denmark \\ ${ }^{\star}$ Corresponding Author. \\ doi: 10.1192/j.eurpsy.2021.124
}

Negative symptoms are a core feature of schizophrenia spectrum disorders associated with poor outcomes such as low remission rates and impairments in daily functioning and quality of life in early psychosis. The assessment of negative symptoms in early psychotic disorders is predominantly conducted by use of firstgeneration scales such as the PANSS and the SANS, along with the SIPS and CAARMS for the psychosis clinical high-risk (CHR) state.
Following the progressed conceptualization of negative symptoms, it has, however, been recognized that these scales suffer important methodological limitations. This warrants a use of secondgeneration scales such as the Brief Negative Symptom Scale (BNSS) and the Clinical Assessment Interview for Negative Symptoms (CAINS) in early intervention settings in order to achieve a more accurate assessment of the negative symptom complex. Advancing the assessment of negative symptoms in early psychosis may also guide more targeted intervention approaches aimed at improving functional outcome. Albeit recognizing that negative symptoms constitute an important barrier to a good functional outcome in psychotic disorders, few studies have directly aimed at alleviating negative symptoms in early psychosis. Meta-analytical evidence does, however, exist on the efficacy of the combined treatment modalities incorporated in Early Intervention Services (e.g. intensive and assertive case management, family involvement etc.) in reducing negative symptoms in first-episode psychosis. Evidence on the effect of interventions for improving negative symptoms in the CHR state is lacking. Developing targeted, and possibly more individualized negative symptoms treatment approaches, constitute an essential future research area.

Disclosure: No significant relationships.

Keywords: Assessment negative symptoms; Negative symptoms early psychosis; Functional outcome early psychosis

\section{S0128}

The challenges in schizophrenia treatment in real-life: The uncomfortable truth

\section{E. Rancans}

Department Of Psychiatry And Narcology, Riga Stradins University, Riga, Latvia

doi: 10.1192/j.eurpsy.2021.125

Certain percentage of the first-episode schizophrenia patients presents with negative symptoms, which persists over the year and influence treatment outcomes (Galderisi et al. 2013). Treatment of negative symptoms has been a significant continuous clinical challenge. Majority of recently published guidelines recommend antipsychotic monotherapy as the standard of care, recommending antipsychotic combination therapy only after a failed trial with clozapine (George A. Keepers et al. 2020; Faden et al. 2020). However, real-life forces clinicians to look for possible combinations of medications early on, especially to tackle negative symptoms. The systematic review of global prescribing practices covering four decades found the pooled median rate of antipsychotic combination therapy approximately $20 \%$ (Gallego et al. 2012). One of the largest retrospective studies every conducted $(n=62,250)$ assessed rehospitalisation rates and the long-term use of antipsychotic polypharmacy in schizophrenia. Antipsychotic combination treatment was associated with an approximate $10 \%$ lower relative risk of psychiatric rehospitalisation compared with antipsychotic monotherapy (Tiihonen et al. 2019). Real-world effectiveness study of antipsychotic monotherapy vs. polypharmacy in schizophrenia from Eastern Europe is also supporting this approach (Katona, Czobor, and Bitter 2014). At the same time antipsychotic combination therapy can increase the total antipsychotic dose burden, frequency of adverse effects, potential drug-drug interactions and incur additional costs. In our recent naturalistic study in schizophrenia outpatients $(n=120)$ with insufficient effectiveness of previous antipsychotics 